\title{
A Throughput and Spectrum Aware Fuzzy Logic Based Routing Protocol for CRN
}

\author{
Poonam Mittal \\ YMCA University of Science and Technology, India \\ E-mail: poonamgarg1984@gmail.com \\ Mehak Jain \\ YMCA University of Science and Technology, India \\ E-mail: mjain870@gmail.com \\ C.K. Nagpal \\ YMCA University of Science and Technology, India \\ E-mail: nagpalckumar@rediffmail.com \\ Shailender Gupta \\ YMCA University of Science and Technology, India \\ E-mail: shailender81@gmail.com
}

\begin{abstract}
Cognitive Radio has been considered a key technology in the future wireless communication. These radio networks with their spectrum sensing capability have become one of the most reliable wireless communication networks. Basically, these networks employ two types of users PU's (Primary Users) having licensed frequency band for their usage and SU's (Secondary Users) that can intelligently detect which communication channels are available and move into vacant ones while avoiding occupied ones. To have communication among these users, a routing protocol is used whose basic purpose is to provide a reliable route from source to destination. An important parameter while designing a routing strategy is the consideration of overall throughput of path. In this paper we consider the same to find the most optimal throughput path using fuzzy logic theory. MATLAB-7.01 is used for implementation and results show that our proposed scheme outperforms shortest spectrum aware routing scheme.
\end{abstract}

Index Terms - Throughput, Cognitive Radio Networks, Routing Protocols, Fuzzy Expert System, Spectrum.

\section{INTRODUCTION}

Cognitive Radios Networks (CRN) [1-4] have got widespread popularity in the recent past due to its spectrum sensing and adaptive capability to select vacant channel while avoiding occupied ones. In spite of this advantage, it has certain limitations too such as limited transmission range, battery power. So, designing a routing protocol that could sense spectrum and avoid its shortcomings is a very challenging task for researchers but still, various routing schemes have been proposed based on various performance metrics in the literature.

\section{RELATED WORKS}

Various routing schemes have been proposed based on performance metrics as follows:

\section{A. Delay}

It is an important performance metric for route establishment. It measures the total time needed to send a data packet from source to destination. Total delay is evaluated by summing all types of delay, i.e., Switching Delay, Transmission Delay, Queuing Delay and Propagation Delay. Multi-hop Single-transceiver CRN Routing Protocol (MSCRP), was the routing protocol where channel and flow scheduling is done at network layer. In Multi-hop Single-transceiver Cognitive Radio Network (MSCRN) [5-8], when a switching node switches to channel $\mathrm{i}$, the LEAVE/JOIN messages have to be sent. This reduces the time available for data transmitting and receiving. The reduced time is named protocol delay and defined as:

$$
\text { Dproto }, i=\text { Dleave }, i+\text { Djoin }, i
$$

Switching between channels would bring switching delay because switching the channel of a wireless transceiver requires changing the input voltage of the VCO, which operates in a Phase Locked Loop (PLL) to achieve the desired output frequency. The delay in channel switching is due to this settling time. This delay is called as Hardware Switch Delay ( $\mathrm{D}_{\text {Hard-Switch }}$ ). The hardware switching delay depends on the relative positions of the two channels on the radio spectrum.

This switching delay is used as routing metric in MSCRN but here channel scheduling is not at every node and it has ignorance towards queuing delay. 


$$
\text { Dswitching }=\text { Dhard }_{-} \text {switch }+ \text { Dproto }, i
$$

Spectrum Aware On-Demand Routing Protocol (SORP) [9] and Delay Metric On-Demand Routing Protocol (DORP) [10] routing scheme works well for Delay sensitive applications.

A Spectrum aware On-demand Routing Protocol (SORP) was the first routing protocol in which Switching Delay (Tsd) between channels and the Back-off Delay (Tbd) within channel were considered to select routes.

$$
T t d=T s d+T b d
$$

Hence by seeing value of the switching delay between different frequency spectrum and back-off delay which occurs due to collisions routing decisions are made.

After that routing and spectrum are considered together and a Delay motivated On-demand Routing Protocol (DORP) was proposed. This joint approach can reach an optimal frequency band switch decision and then select effective routes. In this routing scheduling-based channel assignment progress in CR nodes and find that Queuing Delay (QD) plays an important role in end-to-end delay in CRN.

$$
T t d=T p a t h \_d e l a y+T n o d e \_d e l a y
$$

\section{B. Spectrum}

Spectrum Aware Mesh Routing (SAMER) [11] helps in short term opportunistic utilisation of spectrum. Spectrum Aware Routing Protocol (SPEAR) [12] and Backup Channel and Cooperative Channel Switching (BCCCS) [13] also help in better spectrum management and thus, minimize packet collision. Ant-Based Spectrum Aware Routing (ASAR) [14] based on swarm intelligence has adaptation and self-learning mechanism for spectrum availability. Routing and Spectrum Allocation Algorithm (ROSA) [15] and Weighted Hop, Spectrum Aware and Stability (WHAT) [16] maximize spectrum Utility.

\section{Load Balancing}

Few researchers took load balancing as the performance metric to utilise the network efficiently. Basically, it tries to avoid congestion of a path by using an alternative path in which load is less. The Local Coordination Based Routing [17] protocol is based upon load balancing scheme.

\section{Geographical Location Based}

It is a performance metric which considers the physical topology of a network and adapts itself to change in topology. IP Spectrum Aware Geographic (IPSAG) [18] is a geographical location based routing protocol which is adaptable to change in topology. GC-IPSAG [19] is a gateway cluster IPSAG wherein destination position is known by source. Spectrum Aware Routing Protocol for Cognitive Radio Networks (SEARCH) [20] is a greedy location based routing and Location Aided Routing Protocol for CRN (LAUNCH) [21] is a location based routing protocol which is simple than SEARCH, gives good performance evaluation analysis and also minimizes channel switching delay.

\section{E. Mobility}

One important parameter on which the performance usually depends on is the mobility of the path. More are the mobile nodes in a path, more are the chances of link failures and hence the overall efficiency of network reduces. The Reverse Labelling Instruction (RLR) [22] protocol is based on Spectrum Mobility and provides better link stability and acceptable level of control packet overhead.

\section{F. Hop Count}

It is the number of intermediate nodes between source and destination. Spectrum Aware Routing Protocol for Cognitive Radio Networks (SEARCH) is a greedy location based on this routing strategy that tries to minimize hop count.

\section{G. Transmission Power}

It is the power consumed in a network while transmitting a data packet. It depends on square or fourth power of the Distance between Nodes. Thus, to conserve our battery power, we should pass our data packets to the nearest neighbouring node and so on until it reaches to its destination. Minimum Weight Routing Protocol (MWRP) [23] focuses on Transmission Power and helps in reducing Power Consumption.

In this paper, we have considered throughput to select the most optimal path. The ability of considering the impact of throughput as input parameter by using Fuzzy Logic [24-25] rule base made it more efficient routing scheme. High Throughput is essential for Military Application Networks and multimedia applications. The rest of the paper is organised as follows: Section 3 gives the proposed routing scheme. Section 4 gives the simulation setup parameters, tool used and the snap shot of the simulation process. Section 5 gives the impact on performance metric because of the proposed routing scheme followed by conclusion and references.

\section{THE PROPOSED Routing SCHEME}

Before discussing our proposed routing scheme, we would like to brief you about the fuzzy logic that we have used in order to have a good understanding of our proposed scheme.

\section{A. Fuzzy Logic Controller}

The basic blocks of Fuzzy logic Controller are explained as:-

- Fuzzification Module: This module converts the crisp inputs into linguistic variables. Linguistic variables are defined as per the domain of input variables. Membership functions can be Triangular, Gaussians and Sigmoids etc. 


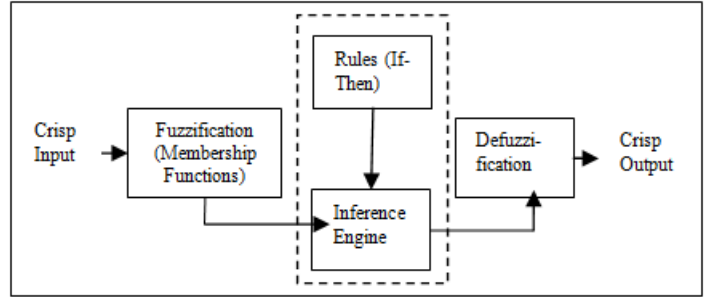

Fig.1. Fuzzy Logic Controller

- Rule Base: This module contains the conditional rules in the form of If-Then and if conditions may have two or more variables connected via And / Or condition. The inference engine works on rule-base. In the rule base, fuzzy inputs are compared with membership function of each input.

- Inference Engine: It evaluates which control rules are relevant at execution time for current input and decides what to display as output. Here, we have used Mamdami- type Inference Engine. This inference engine expects output membership function to be fuzzy sets.

- Defuzzification Module: It is a module which converts the fuzzy outputs back into crisp (quantifiable) results. There are various defuzzification methods available like bisector, centre of area, centroid and centre of gravity etc. But in our proposed method we used Centre of gravity (CoG) method of defuzzification.

\section{B. FLC structure for Shortest Throughput Aware routing protocol}

Figure 2 indicates the block diagram of proposed routing scheme based on throughput as input and optimal path as output. The process for selecting the best possible route can be summarised as:-

- The first step is to compute the throughput of all the spectrum aware possible routes from source to destination.

- The next step is to apply Mamdami - FLC to compute the output weights of all the possible routes.

- Last step is to use this output weight for selecting the optimal path. The path with highest output weight is selected for data transfer.

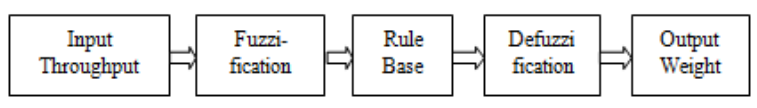

Fig.2. Block Diagram of FLC

The basic blocks of FLC are explained as below:-

- Input to FLC - Throughput: - To compute the optimal route, the FLC uses Throughput as input parameter. It is the rate at which data flows. It varies between different nodes for a particular path, hence minimum data rate is considered as throughput of the overall path (where $\mathrm{k}$ varies from source to destination) as under.

$$
[\text { Throughput }]=\operatorname{Min}[\text { Throughput }] k
$$

- Fuzzification: - The input parameter used in FLC is a crisp value. The fuzzification process converts the crisp data into fuzzy set using membership function as shown in below given figure:-

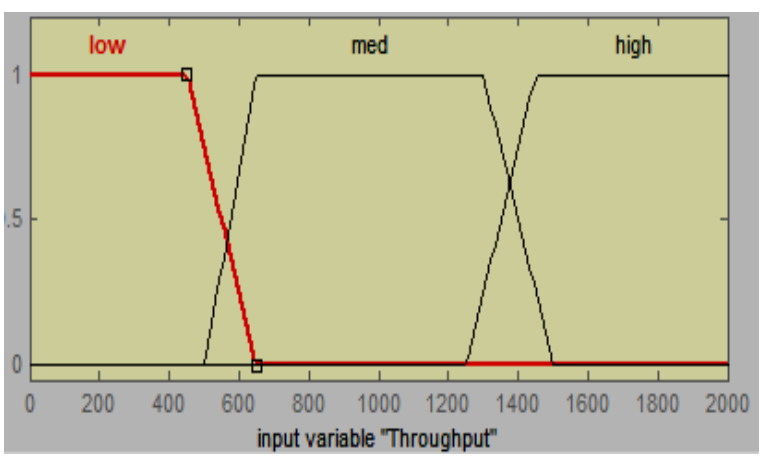

Fig.3. Membership function for Throughput

- Membership Function of FLC Input:-Each of the semantic variables are characterised by membership functions as shown in above figure. Crisp value of input variables is same but their membership function is different. The semantic variable for input parameter is characterized as:-

$$
T(\text { input })=\{[\text { low, medium, high }]\}
$$

Following table 1 is used for fuzzification where Throughput (TH) is mapped as shown in the table 1 and output is obtained as Optimality of Path (OP).

Table 1. Fuzzification of Input as Throughput (TH)

\begin{tabular}{|l|l|l|}
\hline $\begin{array}{l}\text { Membership } \\
\text { Function }\end{array}$ & Optimality of path & Throughput (input) \\
\hline \multirow{3}{*}{ Low } & 1 & $0 \leq \mathrm{TH} \leq 450$ \\
\cline { 2 - 3 } & $3.25-0.05 * \mathrm{TH}$ & $450 \leq \mathrm{TH} \leq 650$ \\
\hline \multirow{3}{*}{ Medium } & $0.0067 * \mathrm{TH}-3.33$ & $500 \leq \mathrm{TH} \leq 650$ \\
\cline { 2 - 3 } & 1 & $650 \leq \mathrm{TH} \leq 1300$ \\
\cline { 2 - 3 } & $7.5-0.005 * \mathrm{TH}$ & $1300 \leq \mathrm{TH} \leq 1500$ \\
\hline \multirow{3}{*}{ High } & $0.005 * \mathrm{TH}-6.25$ & $1250 \leq \mathrm{TH} \leq 1450$ \\
\cline { 2 - 3 } & 1 & $1450 \leq \mathrm{TH} \leq 2000$ \\
\hline
\end{tabular}

- Rule Base for FLC: - Rule base Table is used here by mamdani inference engine to infer the optimality of routes generated from multiple iterations performed by simulator.

1. If (Throughput is high) then (output is best)

2. If (Throughput is med) then (output is moderate)

3. If (Throughput is low) then (output is poor)

- Defuzzification: - It is the process of converting the produced results into crisp outputs through fuzzy logic. The output of FLC is the crisp value evaluated through membership function as shown in the figure. Method used for determining the defuzzified value is $\mathrm{CoG}$ method as shown in the equation:- 


$$
\text { Output }=(m 1 * A 1+m 2 * A 2) /(A 1+A 2)
$$

Where $\mathrm{m} 1$ and $\mathrm{m} 2$ are the membership functions determined through the Fuzzification process as \{Poor, Moderate, Best $\}$ are selected on the basis of rules defined in rule base.

- Membership Function of FLC output: - The output variable of FLC is defined as optimality of the route. The semantic variable for output is characterized as $\{\mathrm{T}$ (output) $\}=\{[$ Poor, Moderate, Best $]\}$. Figure 4 shows that optimality of a path lies between 0 and 1 .

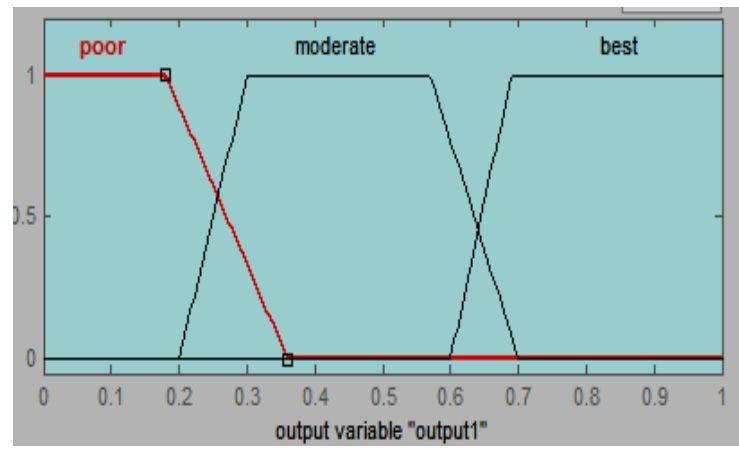

Fig.4. Membership function for Output

Table 2 is used for defuzzification that characterizes the membership functions where Optimality of Path (OP) is used as input and it produce output weight.

Table 2. Fuzzification of Output Weight

\begin{tabular}{|l|l|l|}
\hline $\begin{array}{l}\text { Membership } \\
\text { Function }\end{array}$ & Output Weight & $\begin{array}{l}\text { Optimality of path } \\
\text { (input) }\end{array}$ \\
\hline \multirow{3}{*}{ Poor } & 1 & $0 \leq \mathrm{OP} \leq 0.18$ \\
\cline { 2 - 3 } & $2-5.56 * \mathrm{OP}$ & $0.18 \leq \mathrm{OP} \leq 0.36$ \\
\hline \multirow{3}{*}{ Moderate } & $10 * \mathrm{OP}-2$ & $0.2 \leq \mathrm{TH} \leq 0.3$ \\
\cline { 2 - 3 } & 1 & $0.3 \leq \mathrm{TH} \leq 0.57$ \\
\cline { 2 - 3 } & $5.38-7.69 * \mathrm{OP}$ & $0.57 \leq \mathrm{TH} \leq 0.7$ \\
\hline \multirow{3}{*}{ Best } & $11.11 * \mathrm{OP}-6.67$ & $0.6 \leq \mathrm{TH} \leq 0.69$ \\
\cline { 2 - 3 } & 1 & $0.69 \leq \mathrm{TH} \leq 1$ \\
\hline
\end{tabular}

- Output Weight: - Outputs are assigned some weights and the priority is given to route with maximum weight.

\section{EXPERIMENTAL SETUP}

The proposed QoS based routing protocol based on fuzzy logic controller is implemented in MATLAB and compared with shortest spectrum aware routing protocol exploiting CRN capabilities. This section gives detail of setup parameters, tool used for implementation and snapshots of the proposed scheme implemented.

\section{A. Set-up Parameters}

Table 3. Shows set-up parameters used in our experiment:
Table 3. Set-Up Parameters

\begin{tabular}{|c|c|}
\hline Area & $1500^{*} 1500$ \\
\hline Transmission Range & 400 \\
\hline Nodes(SU) & $24-36$ (Step size 6) \\
\hline Nodes (PU) & 16 \\
\hline Position of SUs & Random \\
\hline Position of Pus & $416 \mathrm{~m} / \mathrm{sec}$ \\
\hline Max velocity & $0 \mathrm{sec}$ \\
\hline Pause time & 25 \\
\hline Data rate (our-actual) & Chosen randomly from SU \\
\hline No of iteration & Chosen randomly from SU \\
\hline Source & 4 \\
\hline Destination & 20 sec \\
\hline No of channels per user/node: & Random walk \\
\hline Simulation Time for 1 & 130 \\
\hline iteration & \\
\hline Mobility Model & \\
\hline Number of Packets sent & \\
\hline
\end{tabular}

\section{B. Performance Metrics used}

- Packet delivery ratio (PDR): It is the ratio of number of data packets delivered to a particular destination to the packets sent from source. Greater value of PDR indicates better performance of a protocol.

- Transmission power: It is the total power consumed to forward data from source to destination [26-28]. The path having minimum required transmission power indicates that nodes are less distant comparatively.

- Delay: It is defined as the time needed to reach a data unit from source to destination. It has 4 constituents which are propagation delay, queuing delay, switching delay and transmission delay [27].

- Hop Count: It is the number of intermediate hops from source to destination in the selected path.

- Throughput: It indicates the data rate or speed of the received data in bits per seconds or data packets per second. Data rate may differ in different nodes in a particular path.

\section{Tools Used}

MATLAB- 7.01 was used for our experimental analysis due to its following key features: It is a high level language and works well for visualization, numerical computation and application development. Libraries are easily accessible. There is no need to mention library for each inbuilt function. The basic data element used is a matrix. A simple integer is a matrix of one row and one column. Several mathematical operations such as cross products, inverse matrices, determinants. Plotting of data is easy and we can change sizes, colours, scales, etc by using interactive graphical tools. Vectorized operation commands are needed instead of for and while loop for adding two arrays. Functionality of MATLAB is expanded by addition of toolboxes which helps in creating plots. Built in graphics helps in visualising data. Most of the mathematical functions are readily available in MATLAB which can greatly reduce the complexity of the program. Functions made in MATLAB can be used to return multiple values. Also, we can produce immediate results by interactively executing commands one at a time. 


\section{Snapshots}

Snapshot of the simulation region is shown in the figure 5. Nodes in blue colour are SU nodes which are randomly placed and also move randomly in the region. Nodes in red colour are PU nodes which are fixed.

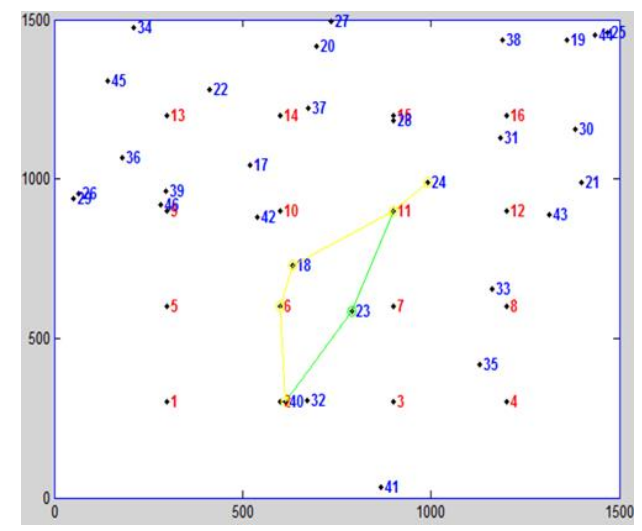

Fig.5. Snapshot of Simulation Process

Green line in the snapshot indicates shortest spectrum aware path from source to destination and yellow line indicate the path obtained from proposed QoS based protocol. In the given snapshot, node 2 is the source and node 24 is the destination.

\section{PERFORMANCE RESUlt COMPARISON}

The algorithm to evaluate the performance of the network in terms of performance metric is described as under. There are 16 nodes placed at fixed locations in the simulation region; each PU node has 4 channels. 25 iterations are performed by increasing the concentrations of SUs from 24 to 36 with a step size of 6 . Multiple iterations are performed to find the efficiency of the system with different pair of source and destination.

Algorithm1. Pseudo-code description of QoS parameter calculation

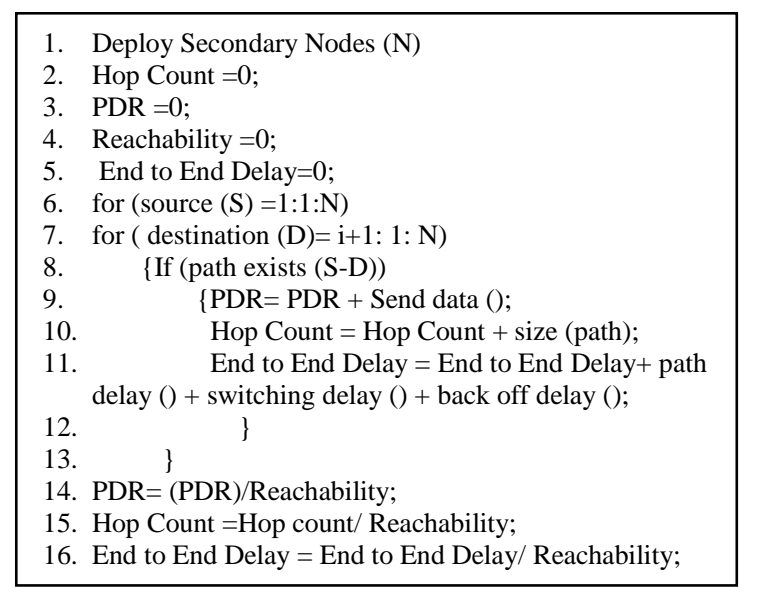

Initially all the nodes are deployed randomly and all the QoS parameters (hop count, PDR, path reach ability and End to end delay are initialized as 0 and after that multiple iterations, 25 in our case, execute on different source to destination pair to find the possible paths and all the QoS parameters are computed for every path and after all iterations, average of all is computed for all QoS parameter. These crisp values of QoS parameters are provided as input to FLC to find the optimal path.

\section{A. Impact on Throughput}

As discussed, our proposed protocol takes into account the value of throughput while shortest spectrum aware consider hop count for route selection hence the value of throughput is much higher in our case as also depicted from the Figure 6. Because less number of hops did not say anything about reliability of path. If the path obtained in shortest spectrum aware routing is reliable (every packet is successfully forwarded to destination) then it has better performance but this reliability is not sensed in shortest spectrum aware routing.

\section{B. Impact on $P D R$}

Figure 7 shows the Average Packet Delivery Ratio (PDR) comparison of Shortest Spectrum Aware and Shortest Throughput Aware Routing Protocol with number of secondary users increasing from 24 to 36 at a step size of 6 . The result shows that PDR is initially higher for shortest spectrum aware routing because when hop count is less then very less packets are dropped and PDR improves. But as the number of secondary users increases (whose performance is dependent on primary users which have fixed count and these secondary nodes starts dropping the packets due to the lack of resources). Hence as the concentration of secondary users increases shortest throughput aware routing starts performing better than shortest spectrum aware routing.

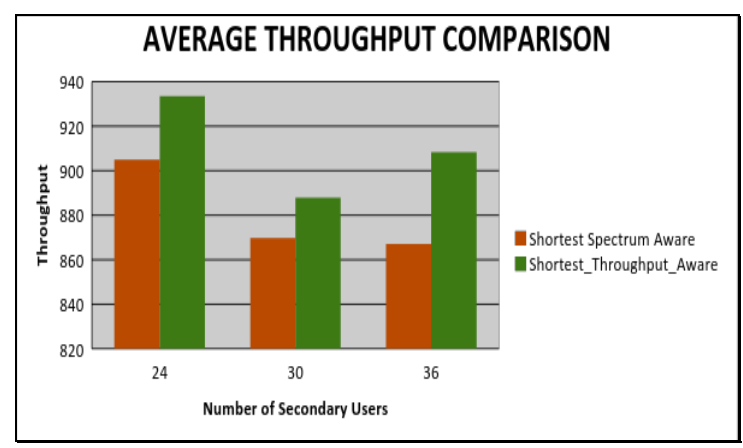

Fig.6. Impact on Throughput

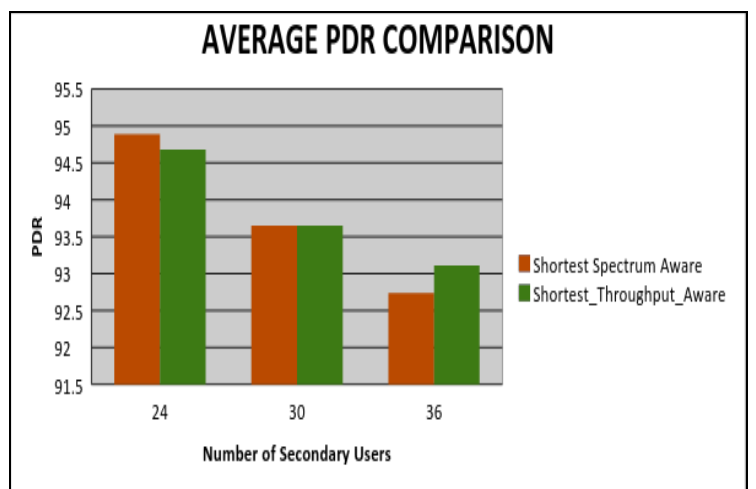

Fig.7. Impact on PDR 


\section{Impact on Hop Count}

Figure 8 shows the comparison of Shortest Spectrum Aware and Shortest Throughput Aware Routing protocol in terms of Hop Count with increasing number of secondary users from 24 to 36 at an interval of 6 . Shortest Spectrum Aware Protocol considers shortest path, hence Hop Count will be minimum at the best equal. When the concentration of secondary users is very less then hop count may be equal in both the cases but with the increase in concentration of secondary users shortest spectrum aware have shortest path in result comparative to shortest throughput aware routing.

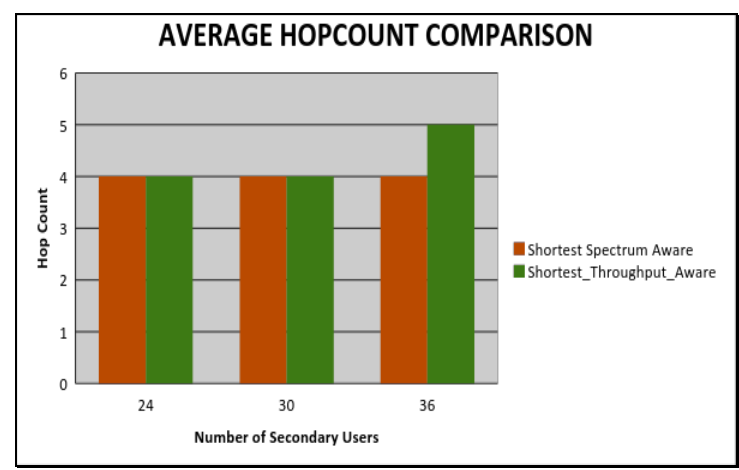

Fig.8. Impact on Hop Count

\section{Impact on Delay}

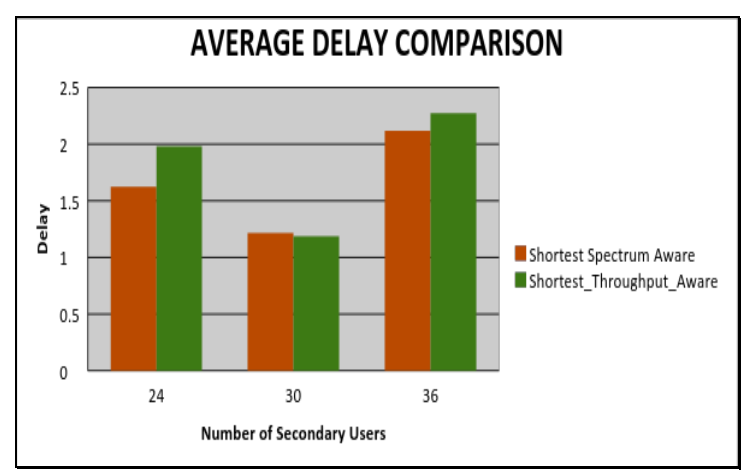

Fig.9. Impact on Delay

Figure 9 shows the comparison of Shortest Spectrum Aware and Shortest Throughput Aware Routing protocol in terms of Delay with increasing Number of secondary users from 24 to 36 at an interval of 6. Shortest Spectrum Aware Protocol considers shortest path, hence minimum Hop Count and Delay while Shortest Throughput Aware is based on maximizing throughput and least significant to Delay.

\section{CONCLUSION}

In this paper we proposed a spectrum aware routing scheme that uses throughput as a performance metric for path selection. It can find application in military application networks and can be used for live streaming. Table 4 shows the overall comparison of the routing scheme with the shortest spectrum aware routing scheme.
The following important inferences can be drawn as follows:

- The protocol has high throughput since it chooses the path which maximises throughput

- The value of packet delivery ratio is almost same for both of the schemes

- Delay and hop count values are higher in case of proposed scheme since the path is elongated since our schemes do not take hop count into account.

Table 4. Overall Comparison Table

\begin{tabular}{|c|c|c|}
\hline Metrics & $\begin{array}{c}\text { Shortest Path } \\
\text { Throughput } \\
\text { Aware Routing } \\
\text { Scheme }\end{array}$ & $\begin{array}{c}\text { Shortest Path } \\
\text { Spectrum } \\
\text { Aware Routing } \\
\text { Scheme }\end{array}$ \\
\hline Throughput & High & Low \\
\hline Delay & High & Low \\
\hline PDR & Same & Same \\
\hline Hop Count & High & Low \\
\hline
\end{tabular}

\section{REFERENCES}

[1] Y.-C. Liang, K. C. Chen and Geoffrey, "Cognitive Radio Networking and Communications: An Overview", Published in IEEE Transactions on Vehicular Technology, vol. 60, no. 7, (2011) September.

[2] S. M. Kamruzzaman, E. Kim and D. G. Jeong, "An Energy Efficient QoS Routing Protocol for Cognitive Radio and Ad Hoc Networks", Published in International Conference on Advance Communication Technology (ICACT), (2011), pp.344-349.

[3] Y.-S. Chen and S. H. Liao, "Spectrum-aware Routing in Discontinuous Orthogonal Frequency Division Multiplexing-based CR Ad Hoc Networks", Published in IET Networks, vol.1, (2012), pp.20-33.

[4] Kartikay Garg, Mehak Jain, Shailender Gupta and Atefeh Hedayati, "Performance Evaluation of Shortest Path Routing Strategy using Cognitive Radios", Published in International Journal of Future Generation Communication and Networking, vol. 8, no. 1 (2015), pp. 37-46.

[5] H Ma, L Zheng, X Ma and Y Luo, "Spectrum aware routing for multi-hop cognitive radio networks with a single transceiver," published in the $3^{\text {rd }}$ International Conference on Cognitive Radio Oriented Wireless Networks and Communications, pp. 1-6, CrownCom2008.

[6] F. Tang, L. Barolli, and J. Li, "A Joint Design for Distributed Stable Routing and Channel Assignment over Multi-Hop and Multi-Flow Mobile Ad Hoc Cognitive Networks", published in IEEE Transactions Industrial Informatics, Vol. 10(2), pp. 1606-1615, 2012.

[7] R. Han and X. Huang, "Reliable link routing in cognitive radio networks," published in the proceedings of 2 nd International Asia Conference on Informatics in Control, Automation and Robotics, vol. 3, pp. 55-58, CAR-2010.

[8] Wang, X. and Kwon, T.T. and Choi, "A multipath routing and spectrum access (MRSA) framework for cognitive radio systems in multi-radio mesh networks," published in the proceedings of ACM workshop on Cognitive radio networks, pp. 55-60, CoRoNet-2009. 
[9] G Cheng, W Liu, Y Li and W Cheng, "Spectrum aware on-demand routing in cognitive radio networks", published in 2nd IEEE International Symposium on New Frontiers in Dynamic Spectrum Access Networks, pp. 571-574, DySPAN-2007.

[10] G Cheng, W Liu, Y Li and W Cheng, "Joint on-demand routing and spectrum assignment in cognitive radio networks", published in the International Conference on Communications, pp. 6499-6503, ICC-2007.

[11] H. Yi Shi and Y.T., "SAMER: Spectrum Aware Mesh Routing in Cognitive Radio Networks", published in the $3^{\text {rd }}$ IEEE Symposium on New Frontiers in Dynamic Spectrum Access Network, pp. 1-5, DySPAN-2008.

[12] A Sampath, L Yang, L Cao, H Zheng and BY Zhao, " High throughput spectrum-aware routing for cognitive radio based ad-hoc networks, "published in the proceedings of 3rd International Conference on Cognitive Radio Oriented Wireless Networks and Communications , pp. , CrownCom-2008.

[13] M Zeeshan, MF Manzoor and J Qadir, "Backup channel and cooperative channel switching on- demand routing protocol for multi-hop cognitive radio ad hoc networks (BCCCS)", published in the proceedings of 6th International Conference on Emerging Technologies, pp. 394-399, ICET-2010.

[14] B. Li, D. Li, Q. H. Wu and H. Li, "ASAR: ant-based spectrum aware routing for cognitive radio networks" , published in the proceedings of IEEE International Conference on Wireless Communications and Signal Processing , pp. 1-5, WCSP-2009.

[15] L. Ding, T. Melodia, S. Batalama, and J. D. Matyjas, "ROSA: Distributed Joint Routing and Dynamic Spectrum Allocation in Cognitive Radio Ad Hoc Networks", published in the proceedings of 12th ACM International conference on modelling analysis and simulation of wireless and mobile systems, pp. 13-20, MSWiM-2009.

[16] J Chen, $\mathrm{H} \mathrm{Li}$ and $\mathrm{J} \mathrm{Wu}$, "WHAT: a novel routing metric for multi-hop cognitive wireless networks", published in the proceedings of $19^{\text {th }}$ Annual Wireless and Optical Communications Conference, pp. 1-6, WOCC-2010.

[17] Z Yang, G Cheng, W Liu, W Yuan and W Cheng, "Local coordination based routing and spectrum assignment in multi-hop cognitive radio networks, "published in Mobile Networks Applications(Springer),vol. 13(1-2), pp. 67-81, 2008.

[18] C.-I. Badoi, V. Croitoru, and R. Prasad, "IPSAG: An IP Spectrum Aware Geographic Routing Algorithm Proposal for Multi-hop Cognitive Radio Networks," published in the 8th International Conference on Communications, pp. 491-496, COMM-2010.

[19] Cornelia-Ionela Badoi, Victor Croitoru, Neeli Prasad and Ramjee Prasad," HC-IPSAG and GC-IPSAG Algorithm Proposals: Cluster based IPSAG Algorithm Variations for large Cognitive Radio Networks", published in the 52nd International Symposium, pp. 239-242, ELMAR-2010.

[20] R. Chowdhury and M. Di Felice, "SEARCH: A routing protocol for mobile cognitive radio ad-hoc networks", published in the IEEE Sarnoff Symposium, pp. 1-6, SARNOFF-2009.

[21] K. Habak, M. Abdelatif, H. Hagrass, K. Rizc, and M. Youssef, "A Location-Aided Routing Protocol for Cognitive Radio Networks ", published in the proceedings of International Conference on Computing, Networking and Communications, pp. 729-733, ICNC2013.

[22] R. Han and X. Huang, "Reliable link routing in cognitive radio networks," published in the proceedings of 2nd International Asia Conference on Informatics in Control, Automation and Robotics, vol. 3, pp. 55-58, CAR-2010.

[23] M. H. C.W. Pyo, "Minimum Weight Routing based on A Common Link Control Radio for Cognitive Wireless Ad Hoc Networks," published in the International Conference on Wireless Communications and Mobile Computing, pp. 399-409, IWCMC-2007.

[24] Shailender Gupta, Bharat Bhushan and Vivek Kukreja, "A Fuzzy based routing Protocol for Cognitive Radio Networks", published in International Journal of Fuzzy Computation and Modelling (Inderscience-2014).

[25] J. Deng, Y.S. Han, P.-N. Chen and P.K. Varshney, "Optimal Transmission Range for Wireless Ad Hoc Networks Based on Energy Efficiency", published in IEEE Transactions on Communications, vol. 55(9), pp. 1772-1782, 2007.

[26] Omar Smail, Zoulikha Mekkakia, Belhadri Messabih, Rachida Mekki, Bernard Cousin, "Energy Conservation for Ad Hoc On-Demand Distance Vector Multipath Routing Protocol", published in IJCNIS-2014, pp. 1-8, DOI: 10.5815 .

[27] B.Ruxanayasmin, B.Ananda Krishna, T.Subhashini, "Minimization of Power Consumption in Mobile Adhoc Network" published in IJCNIS-2014, pp. 38-44, DOI: 10.5815 .

[28] Vivek Kukreja, Shailender Gupta, Bharat Bhushan and Chander Kumar, "Towards Performance Evaluation of Cognitive Radio Networks in Realistic Environment", published in IJCNIS-2014, pp. 61-77, DOI: 10.5815.

\section{Authors' Profiles}

Poonam Mittal received her B.Tech. and M.Tech. from YMCA University of Science and Technology, Faridabad, India. She is currently working as an Assistant Professor in Computer Engineering Department in the same university. She is currently pursuing Ph.D. Her interests include networking and algorithm design.

Mehak Jain, student of B.Tech. in Electronics Engineering Department at YMCA University of Science and Technology, Faridabad, India. Her interest is in the field of networking.

C.K. Nagpal, Professor and Ph. D. supervisor in Computer Engineering Department in YMCA University of Science and Technology, Faridabad, India. His interests include networking and fuzzy expert system. He has published more than 25 papers in various national and international publications.

Shailender Gupta, received his B.Tech., M.Tech. and $\mathrm{PhD}$ from YMCA University of Science and Technology, Faridabad, India. He is currently working as an Assistant Professor in Electronics Engineering Department in the same university. His interests include networking and image processing. He has published more than 40 papers in various national and international publications. He is also an editor of IJCNIS. 